\title{
DEUS EXISTE? \\ UM ESTUDO PROBABILÍSTICO
}

\author{
Charles Florentino da Silva
}

RESUMO: O presente artigo tem por finalidade a investigação acerca da possibilidade da existência de Deus através de um olhar probabilístico, para isso será apresentado os conceitos da proporção áurea presentes na natureza, seguidas de deduções probabilísticas a cerca desse fenômeno.

Palavras-chave: probabilidade. Matemática. Deus. razão áurea. fenômeno.

ABSTRACT: The purpose of this article is to investigate the possibility of the existence of God through the probabilistic, for which the concepts of the golden ratio present in nature will be presented, followed by probabilistic deductions about this phenomenon.

Keywords: probability. Mathematics. God. reason.

\section{INTRODUÇÃO}

Fé e ciência são assuntos que nos dias atuais não podem coexistir dentro de um mesmo contexto, isto porque a ciência se preocupa em ser exata na interpretação de fenômenos da natureza, bem como no entendimento dos mesmos, em contra partida, a fé é uma característica que varia de indivíduo para indivíduo, não é experimental, sendo assim, não é objeto de estudos da ciência, todavia existe uma proposta cientifica chamada Design inteligente que afirma a possibilidade da verificação da existência de Deus através da observação de fenômenos da natureza, sendo assim, o presente artigo irá averiguar a veracidade de tal afirmação através do pensamento probabilístico aplicados a razão áurea.

\section{OBJETIVO GERAL}

O presente artigo possui como objetivo geral fazer a verificação matemática da influência do "acaso" no funcionamento do universo. 


\section{OBJETIVO ESPECÍFICO}

O Objetivo Específico do presente trabalho tem por finalidade o estudo do fenômeno matemático "razão áurea" através dos critérios de probabilidade, a fim de entendermos se existe a possibilidade de haver ou não uma intencionalidade na criação e funcionamento do universo.

\section{JUSTIFICATIVA}

O Presente trabalho se justifica diante do grande contraste existe entre fé e ciência, sendo assim entende-se como necessário a averiguação cientifica da possibilidade da existência de um "arquiteto" do universo.

\section{PROBLEMA}

Como a matemática pode ajudar a entender se existe ou não um "projetista” para a criação e funcionamento do universo?

\section{CAPÍTULO I: A PROPORÇÃO ÁUREA}

A Proporção áurea (ou razão áurea) é uma proporção relacionada com a beleza, e que se encontra presente nos mais variados cantos da terra e até mesmo do universo. Segundo

LAURO, dês do século III AC já havia relatos de tal fenômeno, que é objeto de estudo até os dias atuais. (LAURO, 2005, p. 36).

\section{DEMONSTRAÇÃO ALGÉBRICA}

Observe o retângulo abaixo de dimensões a e b.

Imagem I

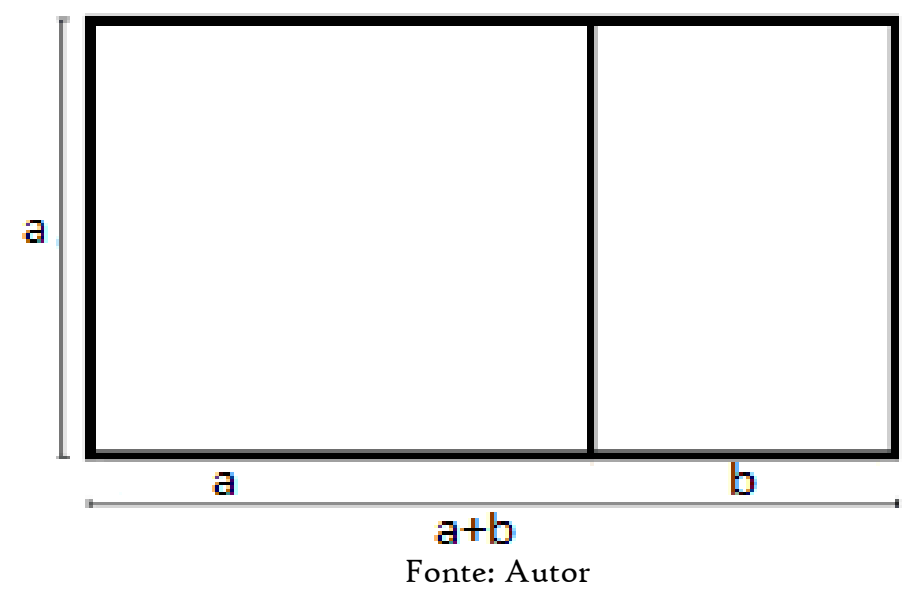


Para o retângulo ter as propriedades da razão áurea, é necessário que:

$\frac{a+b}{a}=\frac{a}{b}$

$a^{2}=a b+b^{2}$

$a^{2}-a b-b^{2}=0$

Dividindo ambos os membros da igualdade por $b^{2}$ temos:

$\frac{a^{2}}{b^{2}}-\frac{a b}{b^{2}}-\frac{b^{2}}{b^{2}}=\frac{0}{b^{2}}$

Se chamarmos a razão $\frac{\mathrm{a}}{\mathrm{b}}=\varphi$ (lê-se "fì"), obtemos a seguinte equação do segundo grau:

$$
\varphi^{2}-\varphi-1=0
$$

Aplicamos então a fórmula de Bhaskara:

$$
\begin{aligned}
\varphi & =\frac{-(-1) \pm \sqrt{(-1)^{2}-4 * 1 *(-1)}}{2 * 1} \\
\varphi & =\frac{1 \pm \sqrt{5}}{2}
\end{aligned}
$$

Como geometricamente não é possível representar uma medida negativa, iremos admitir apenas o valor positivo da equação, portanto:

$$
\begin{gathered}
\varphi=\frac{1+\sqrt{5}}{2} \\
\varphi \cong \mathrm{I}, 6 \mathrm{I} 8
\end{gathered}
$$

Segundo LAURO, pegando o retângulo áureo da imagem I podemos fazer um novo retângulo, que se construído dentro da proporção $\varphi$, terá as mesmas característica do retângulo inicial, podendo assim ser repetido infinitas vezes. (LAURO, 2005, p.40). Dentro do padrão estabelecido, é possível criar uma espiral conforme a figura a seguir: Imagem 2: Espiral Aurea

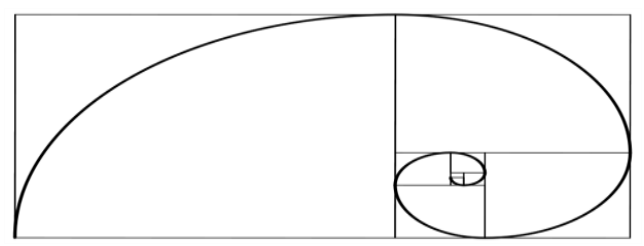

Fonte: LAURO, 2005, p. 40. 


\section{A INFLUÊNCIA DA RAZÃO ÁUREA EM OBRAS ARTÍSTICAS}

A proporção áurea, conforme já mencionada, é objeto de estudos do ser humano a mais de 2300 anos, e influenciou diversos trabalhos artísticos, segue abaixo algumas dessas obras. Imagem 3: Monalisa

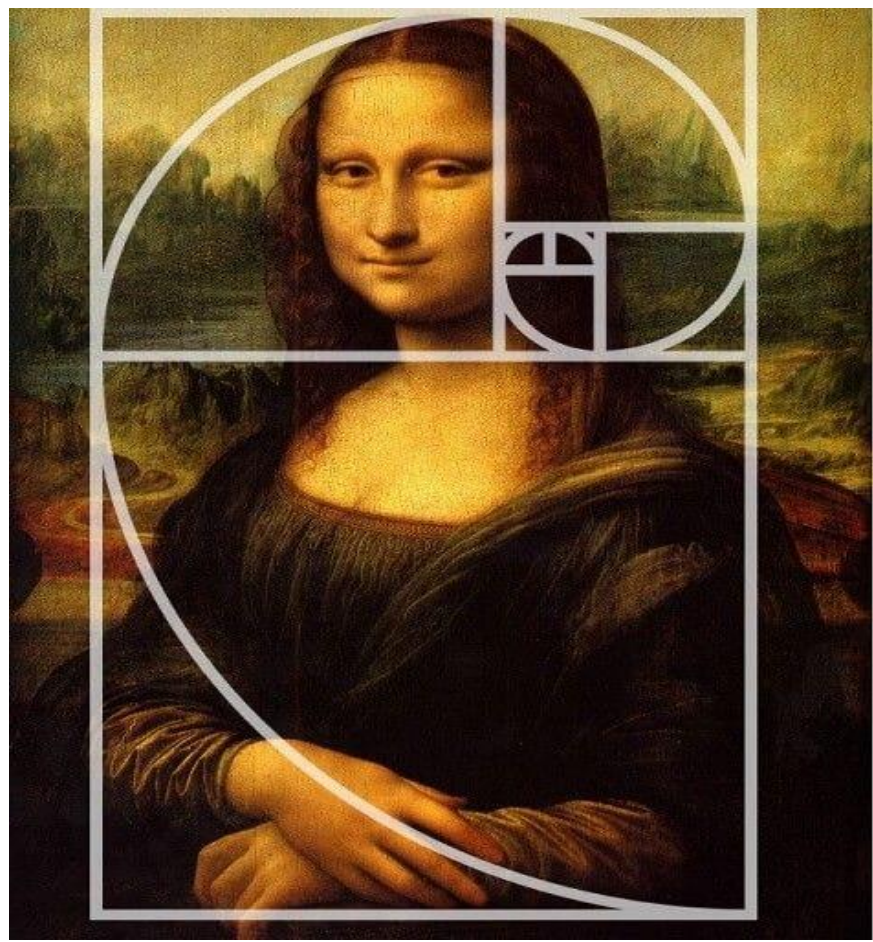

Fonte: https://br.pinterest.com/pin/362469470008257343/

Imagem 4: Fachada de Parthenon.

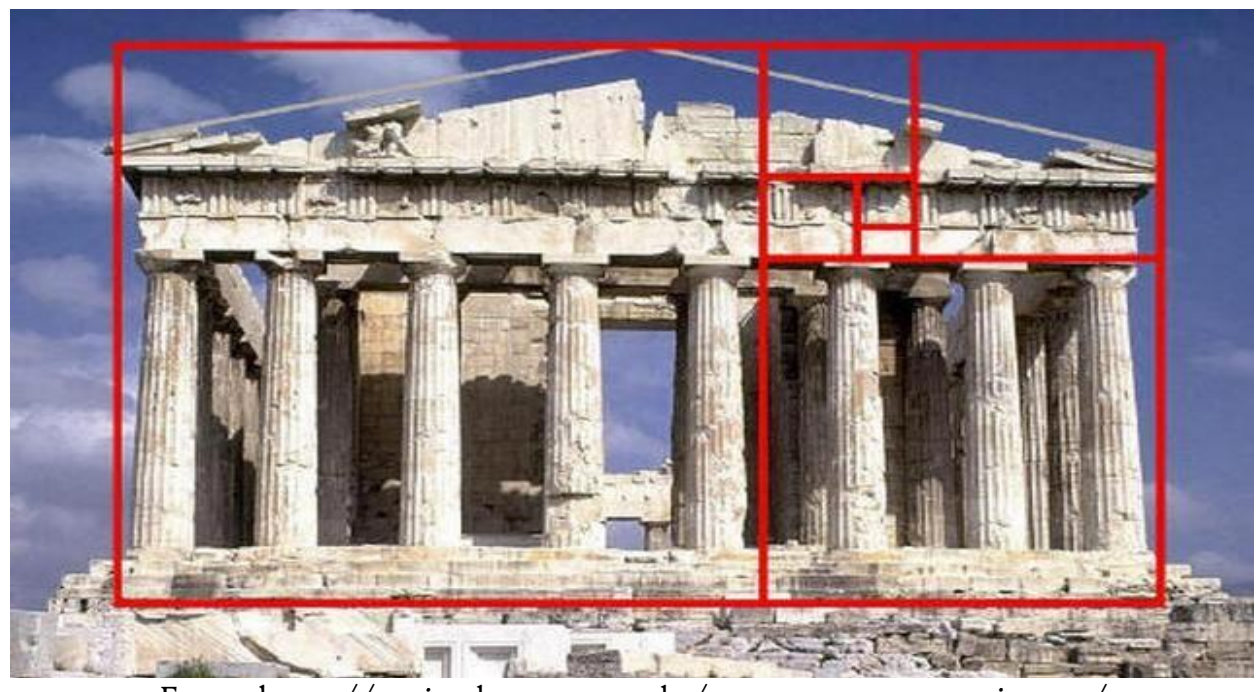

Fonte: https://projetobatente.com.br/regra-aurea-na-arquitetura/ 
Imagem 5: Fachada da Catedral de NotreDame

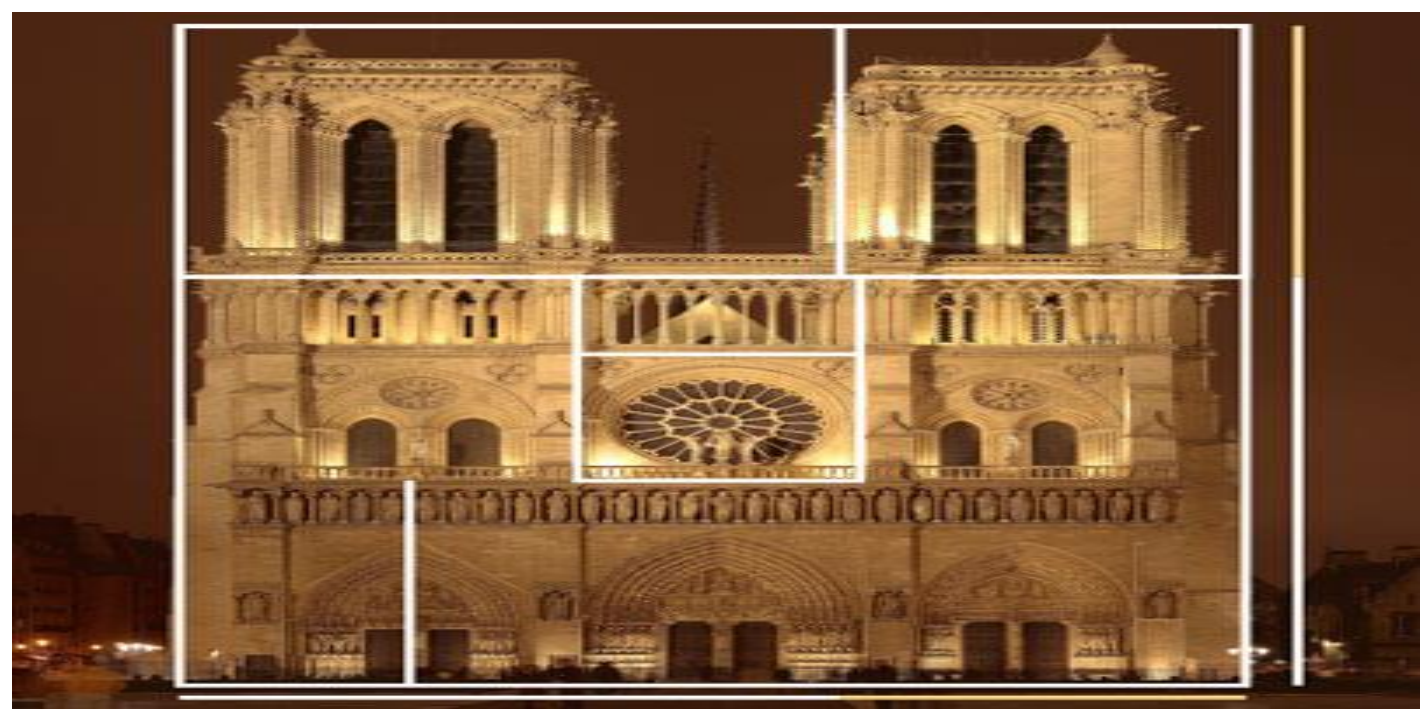

Fonte: https://projetobatente.com.br/regra-aurea-na-arquitetura/

\section{A RAZÃO ÁUREA NA NATUREZA}

A proporção $\varphi$ também é encontrada na natureza, e para esse fenômeno iremos aplicar os conceitos de probabilidade, a fim de averiguar se tal comportamento da natureza poderia ou não ser oriundo de uma "mente inteligente".

A referida proporção é presente em diversas partes da natureza, como nas arvores, nos animais e até mesmo no ser humano (LAURO, 2005, p.44).

\section{Imagem 6 : Árvores}

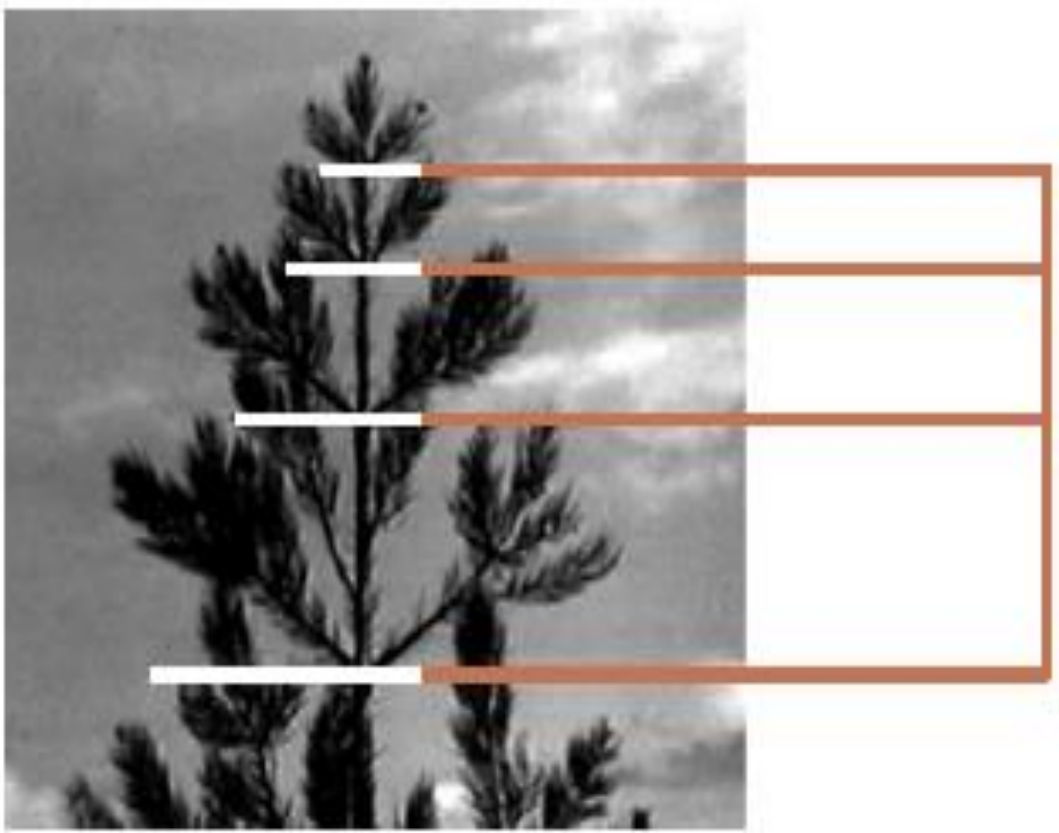

Fonte: ROCHA aput LAURO, p.47 


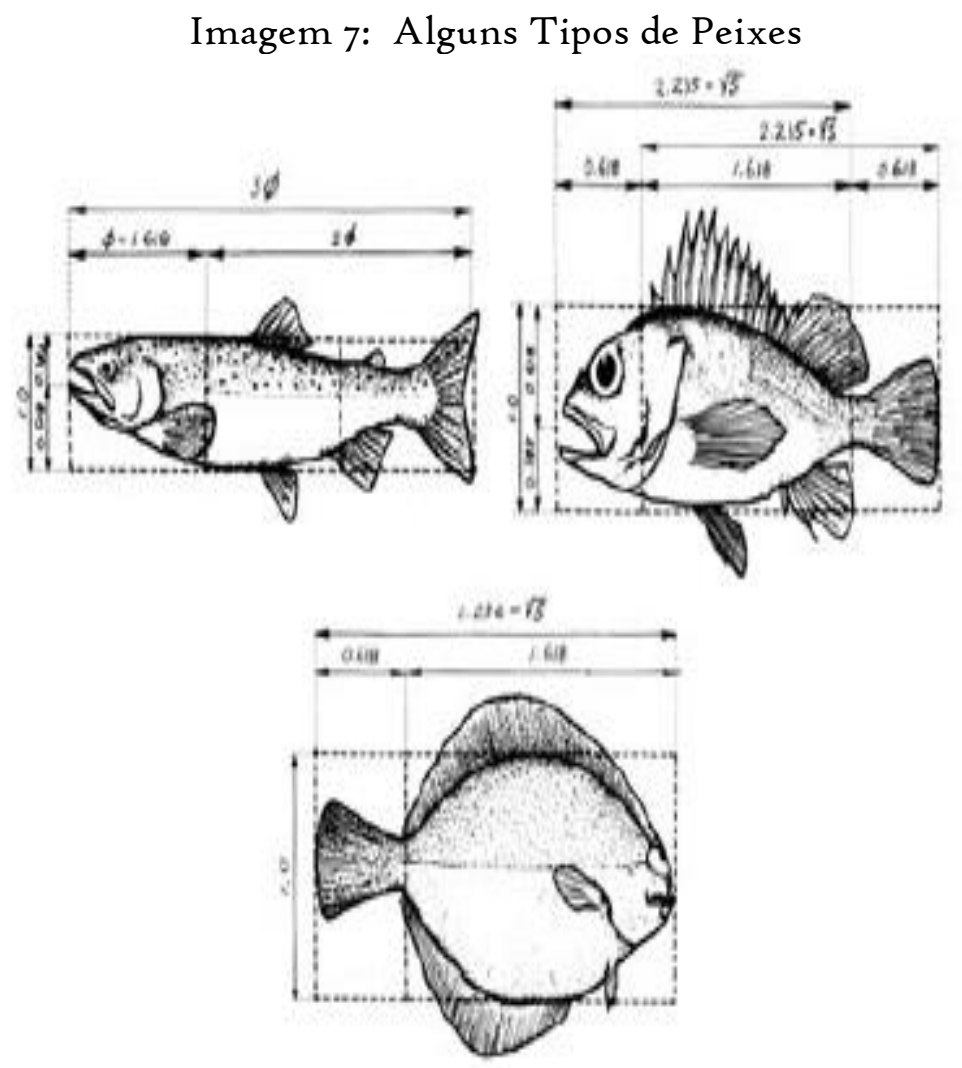

Fonte: ROCHA aput LAURO, p.47.

Imagem 8: Concha do Molusco Nautilus

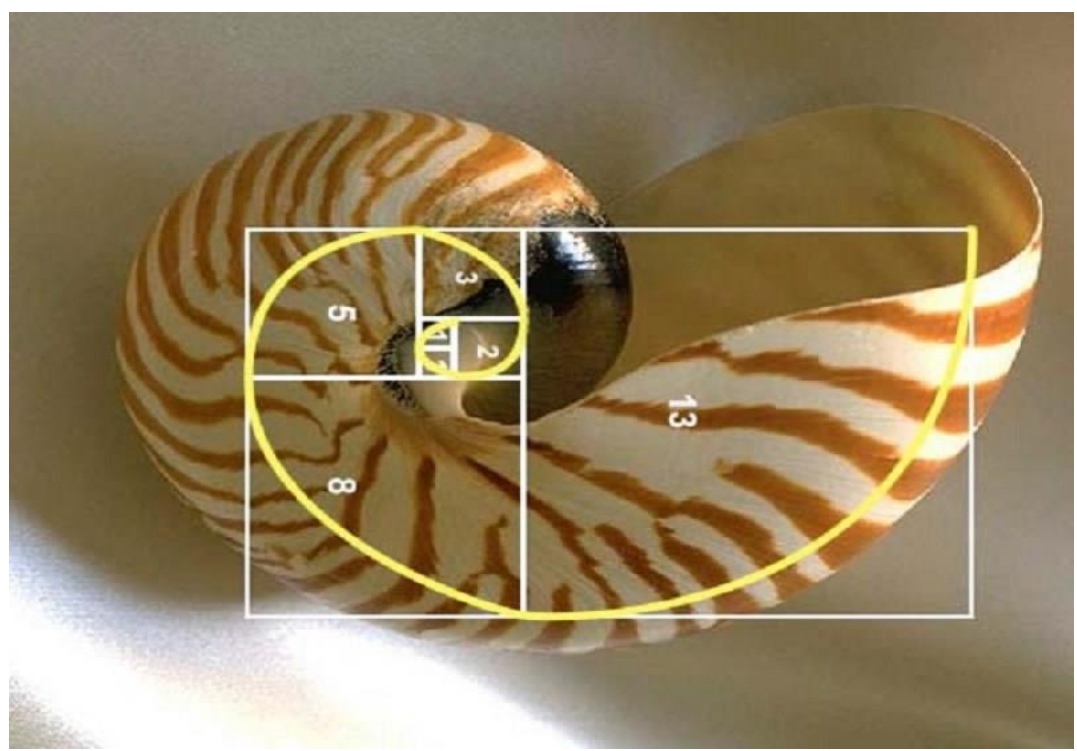

Fonte: https://www.raciociniocristao.com.br/2014/o5/digitais-criador/ 


\section{Imagem 9: Ondas do mar}

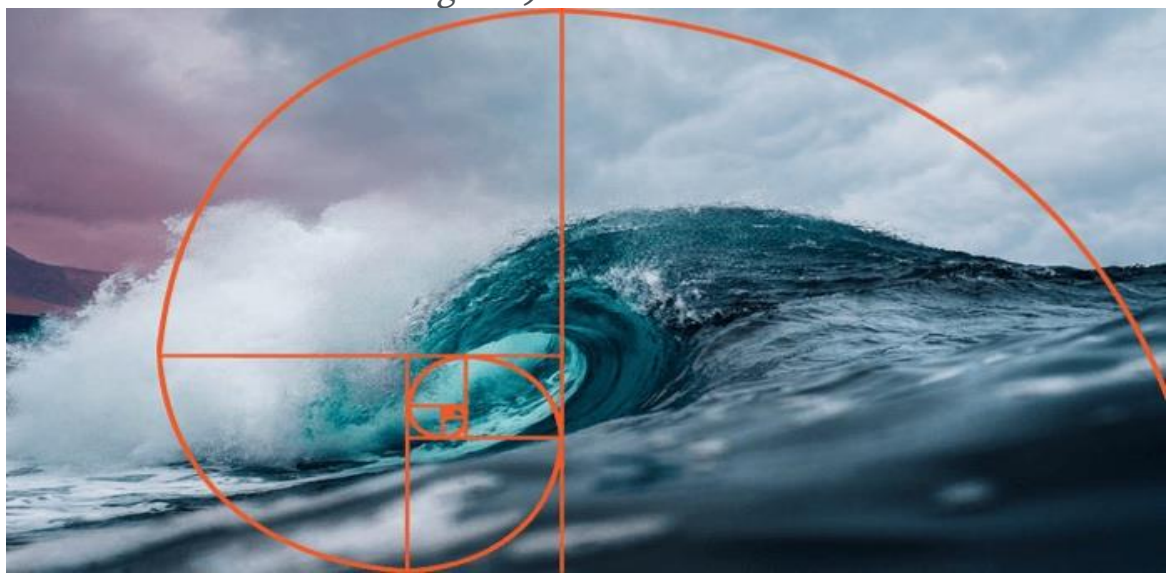

Fonte: https://iphotochannel.com.br/proporcao-aurea-vs-regra-dos-tercos-qual-e-a-melhor-para-comporsuas-fotos/

Imagem ro: Arcada Dentaria

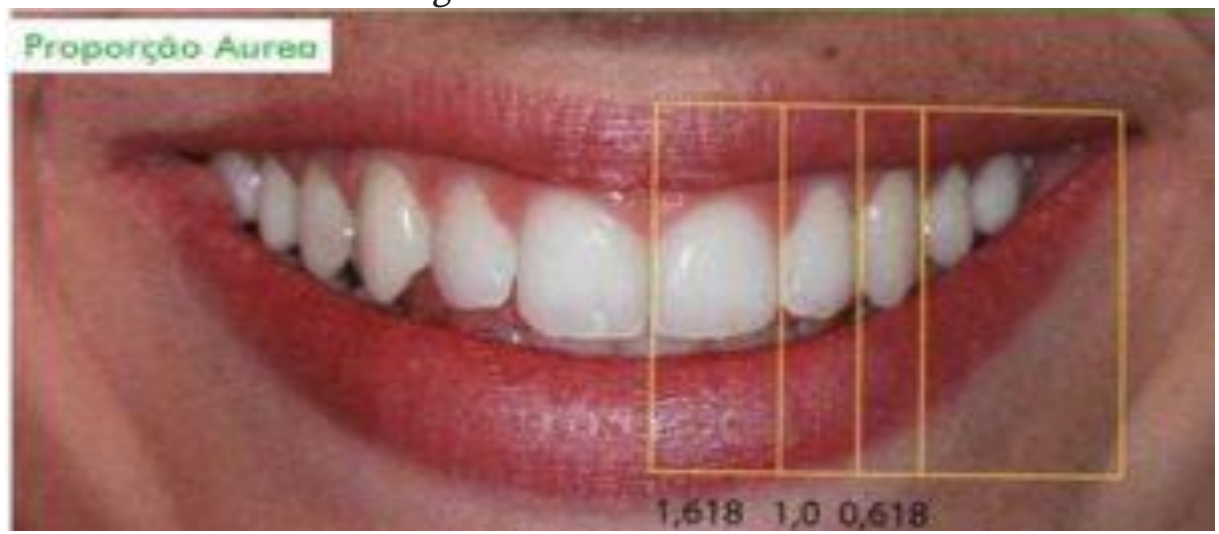

Fonte: http://marcodevilla.com.br/proporcao-e-simetria-dos-dentes-superiores-na-estetica-do-sorriso/

Imagem II: Face dos seres humanos

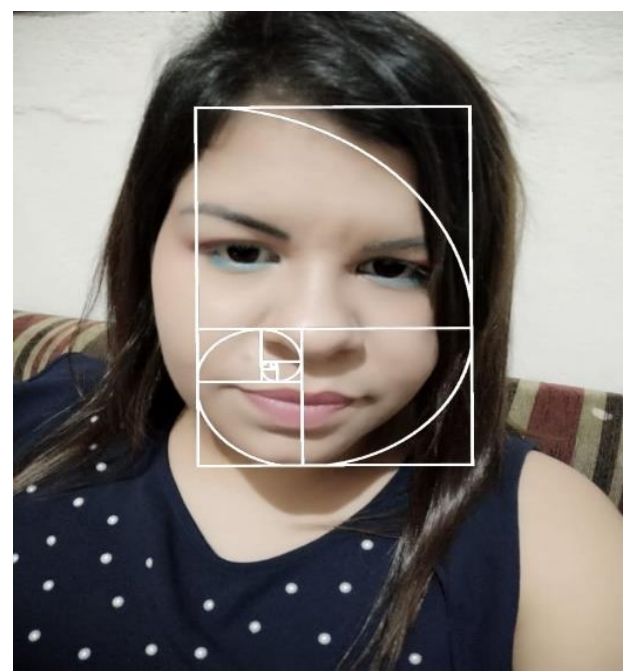

Fonte: Autor 


\section{CAPÌTULO II - PROBABILIDADE}

Probabilidade é o segmento da matemática que estuda a chance de ocorrer um determinado evento de forma aleatória, isto é, sem nenhuma interferência externa. "A teoria das probabilidades é o ramo da matemática que cria, elabora e pesquisa modelos para estudar fenômenos aleatórios”. (DANTE, 2009, p. 299).

\section{EXPERIMENTO ALEATÓRIO}

Segundo Paiva, 2009, pode-se chamar de experimento aleatório todo fenômeno cujo resultado depende unicamente do acaso.

\section{ESPAÇO AMOSTRAL}

Paiva, 2009 entende que por espaço amostral como sendo o conjunto de todos os resultados possíveis de um experimento aleatório.

\section{ESPAÇO AMOSTRAL EQUIPROVÁVEL}

É um conjunto de eventos onde não existe a preferência por nenhum resultado, isto é, todos os eventos possíveis têm a mesma chance de ocorrerem.

\section{EVENTO}

Dante, 2005 entende que por evento qualquer subconjunto de um espaço amostral.

\section{DEFINIÇÃO}

Probabilidade é a razão entre o número de eventos que se está estudando e o total de possibilidades que o espaço amostral oferece. Sendo assim temos:

$$
P(A)=\frac{n(A)}{n(E)}
$$

Onde $\mathrm{n}(\mathrm{A})$ é o número de eventos em estudo (eventos favoráveis), $\mathrm{n}(\mathrm{E})$ é o número de eventos de um espaço amostral, e $\mathrm{P}(\mathrm{A})$ é a probabilidade (chance) de ocorrer o referido evento.

OBS: Para a validação da fórmula descrita, é necessário que o espaço amostral seja finito, não vazio e equiprovável.

Todo cálculo de probabilidade compreende valores que variam entre o e $\mathrm{I}$, sendo que, quanto mais próximo de o, menor a chance de acontecer um dado evento, em contrapartida, quanto mais próximo de I, maiores serão as chances desse evento se realizar. 


\section{APLICAÇÃO PRÁTICA}

Para ilustrar uma aplicação dos conhecimentos de probabilidade, iremos resolver o seguinte exercício extraído de PAIVA: "No lançamento de um dado, qual a probabilidade de se obter, na face voltada para cima, um número menor que 3?”. (PAIVA, 2009, p. 266). Para resolver esse exercício, é necessário conhecer o espaço amostral E, sendo ele composto por todas as possibilidade de resultados que o dado permite, isto é, $E=\{1,2,3,4,5,6\}$, em seguida vamos criar o subconjunto A, formado pelos eventos favoráveis ao estudo, ou seja, $\mathrm{A}=\{\mathrm{I}, 2\}$. Como $\mathrm{n}(\mathrm{E})=6$ (número de elementos do espaço amostral) e $\mathrm{n}(\mathrm{A})=2$ (número de elementos do subconjunto A), temos:

$P=\frac{2}{6} \cong 0,33$

Para melhorar a interpretação dos cálculos, iremos colocar a resposta em forma de porcentagem:

$0,33 * 100=33 \%$

Sendo assim, temos aproximadamente $33 \%$ de chance para que a condição estabelecida ocorra, note que temos um valor abaixo de 50\%, portanto, pouco provável de ocorrer quando comparado com a chance de se obter as demais faces do dado em um lançamento. Observe agora a tabela abaixo, extraída de DANTE, quem contém informações a cerca de um dado lançado 1200 vezes.

Tabela I

\begin{tabular}{|l|l|l|}
\hline Face & $\begin{array}{l}\text { Frequência absoluta (número de } \\
\text { vezes que cada face foi obtida) }\end{array}$ & $\begin{array}{l}\text { Frequência relativa (porcentagem } \\
\text { da frequência Absoluta) }\end{array}$ \\
\hline I & 248 & $20,7 \%$ \\
\hline 2 & 355 & $29,6 \%$ \\
\hline 3 & 175 & $14,6 \%$ \\
\hline 4 & I80 & $15 \%$ \\
\hline 5 & I26 & $10,5 \%$ \\
\hline 6 & I16 & $9,7 \%$ \\
\hline
\end{tabular}

Fonte: DANTE, 2009, p. 33I. 
De acordo com a fórmula apresentada para o cálculo de probabilidade, temos que a chance de sair uma face qualquer desse dado é dada por:

$P(A)=\frac{n(A)}{n(E)}=\frac{1}{6} \cong 0,16$

Em porcentagem teremos:

$0,16 * 100=16 \%$

Ao analisar a tabela observa-se que o número de vezes que as faces i e 2 foram obtidas (20,7\% e 29,6\% respectivamente) é consideravelmente superior aos i6\% que foi previsto, tal observação sugere que o dado não seja totalmente honesto. (DANTE, 2009, p. 331).

A partir dessa análise, é plausível o raciocínio de que um experimento aleatório repetido por uma quantidade considerável de vezes, sempre terá seus resultados dentro do valor teórico previsto, e quando ocorre certa discrepância entre esses valores, existe alguma influência no experimento que não é oriunda do acaso, ou seja, uma interferência intencional.

\section{CAPÍTULO III - INVESTIGAÇÃO E ANÁLISE DAS INFORMAÇÕES}

Uma vez já estabelecidos os conceitos apresentados, o presente capítulo irá fazer uma investigação a fim de responder à pergunta proposta do início do artigo: Como a matemática pode ajudar a entender se existe ou não um "projetista" para a criação e funcionamento do universo?

Façamos o seguinte experimento, observe a malha representada na figura abaixo, contendo 90 quadrados onde cada quadrado representa uma unidade, sendo 9 na horizontal, e io na vertical.

Imagem I2: Malha de Quadrados

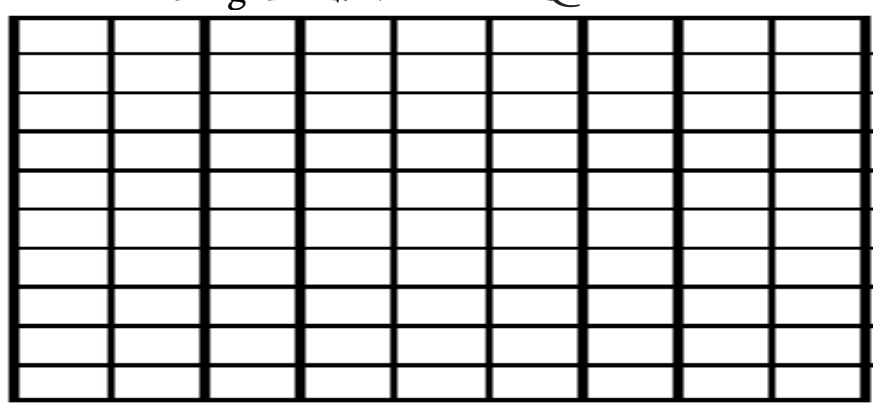

Fonte: Autor 
De acordo com as dimensões fornecidas, apenas um retângulo de $5 \mathrm{X} 8$, com perímetro de 26 unidades obedeceria de forma aproximada o padrão áureo de i,6i8.

Dentro desse contexto, qual seria a probabilidade, de uma pessoa "ao acaso" pintar 26 quadradinhos de forma aleatória, e como consequência obter um retângulo que obedece a proporção $\varphi$ ?

Para responder essa pergunta, a fim de verificar a capacidade do acaso de gerar esse padrão precisamos determinar o número de eventos favoráveis ao experimento, e em seguida determinar o espaço amostral.

Com o auxílio do software "scratch", verificou-se que o número de retângulos áureos que podem ser construídos dentro da malha corresponde a um total de 27.

Observe abaixo o exemplo de uma das 27 posições diferentes que o retângulo pode ser construído:

Imagem 13: Retângulo áureo

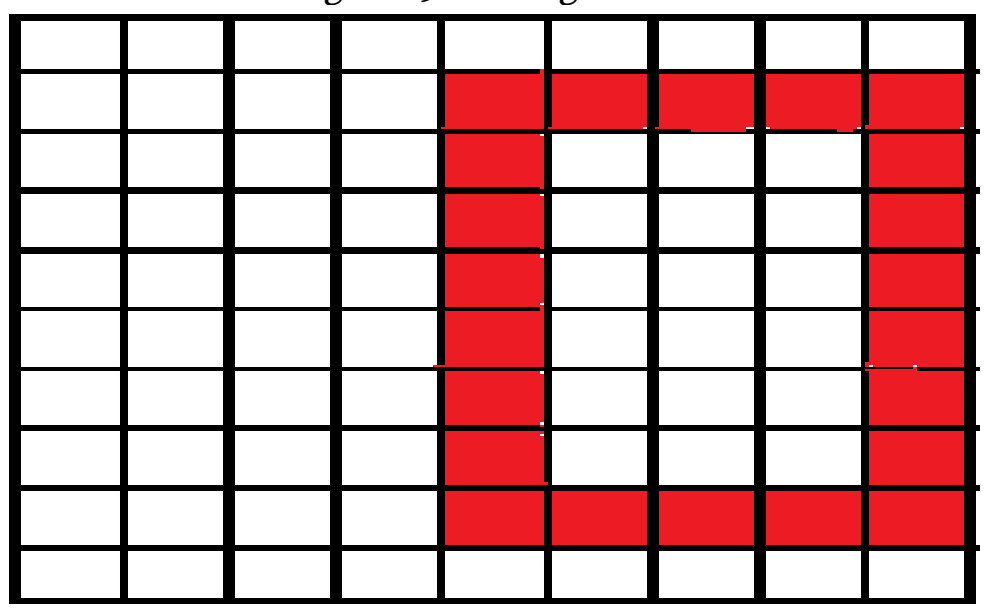

Fonte: autor

Agora é necessário determinar o espaço amostral, isto é, o número de combinações

diferentes que podem ser feitas dentro da malha a partir do preenchimento de 26 quadrados, para isso usaremos a fórmula de combinação simples.

$C n, p=\frac{n !}{(p !(n-p) !)}$

Sendo $\mathrm{C}$ = combinações possíveis e sem repetição, $\mathrm{n}=$ total de elementos disponíveis para as combinações, e $\mathrm{p}$ = número de elementos que se deseja agrupar.

Onde temos: 
$C 90,26=\frac{90 !}{26 !(90-26) !}=29033531588150684937045=\mathrm{CP}$ (combinações possíveis)

Aplicando a fórmula de probabilidade, temos:

$\mathrm{P}=\frac{27}{C P} \cong 0,000000000000000000093$

Em porcentagem teremos:

$0,00000000000000000000093 * 100=0,000000000000000000093 \%$

Embora a probabilidade da criação de um retângulo com os padrões áureos seja pequena, deve-se considerar o tempo que o universo possui para fazer as "combinações necessárias". Segundo CHOI, a idade do universo gira em torno de 14 bilhões de anos. (CHOI, 2020). Ao transformar esse tempo em segundos temos:

I ano tropical $=365$ dias, 5 horas, 48 minutos e 46 segundos

I ano sideral $=365$ dias, 6 horas, 9 minutos e ro segundos

I dia $=24$ horas

$\mathrm{I}$ hora $=60$ minutos $=3600$ segundos

I minutos $=60$ segundos

Portanto, para a quantidade de segundos que teremos em um ano tropical, efetuamos:

$365 * 24 * 3600+5 * 3600+48 * 60+46=31556926$ segundos

Logo para o tempo do universo em segundos, teremos:

$1400000000 *^{*} 31556926=441796964000000000=$ TAT $($ tempo em anos tropicais $)$

Para a determinação dos segundos, de acordo com o ano sideral, calcula-se:

$365 * 24 * 3600+6 * 3600+9 * 60+10=31558150$ segundos

Portanto:

$14000000000 * 31558150=441814400000000000=$ TAS $($ tem em anos siderais $)$

Verifica-se que CP > TAT e CP > TAS.

Constata-se que se cada combinação da malha fosse feita ao acaso a cada I segundo o tempo de existência do universo (independente de considerarmos anos siderais ou tropicais), não seria suficiente para se completar as 27 combinações possíveis.

Os resultados observados corroboram as afirmações de DEMBSKY, de que a probabilidade do acaso gerar informações complexas é extraordinariamente pequena. (DEMBSKY aput BRAGA 2016, P. 93). 


\section{OBSERVAÇÕES}

-Admitindo o tempo de 14 bilhões de anos para o universo, estamos de certa forma "favorecendo" o acaso, uma vez que a maioria das literaturas traz um valor inferior, de aproximadamente 13,8 bilhões de anos.

- Ao pedir para alguém pintar 26 quadrados aleatoriamente, também estamos "favorecendo" o acaso, uma vez que outra quantidade de quadrados a serem preenchidos na malha, não iria produzir um retângulo áureo.

- Ao limitar o tamanho da malha a 90 quadrados, estamos novamente "contribuindo" com o acaso, uma vez que malhas com uma quantidade maior de quadrados embora pudesse conter mais retângulos áureos, produziriam também um aumento exponencial no espaço amostral, o que reduziria ainda mais a probabilidade de que o evento ocorra.

\section{CONCLUSÃO}

Analisando os resultados do experimento, e diante da variedade de fenômenos naturais que apresentam a proporção $\varphi$, e considerando a baixa probabilidade de o acaso gerar padrões complexos, torna-se aceitável e coerente a proposta de um "projetista inteligente" na estruturação do universo.

\section{REFETENCIAS BIBLIOGRAFICAS}

AMADOR, Fabiana dos Santos, Números de Fibonacci, 2013.

BRAGA, Lucas; Entre a Fé e a Ciência: Uma Análise sobre a Teoria do Design Inteligente, 2016.

DANTE; Luiz Roberto, volume único: Livro do Professor, red, São Paulo; Ática. 2009.

LAURO, Maira Mendias; A razão áurea e os padrões harmônicos na natureza, artes e arquitetura, EXACTA. Num3. 2005 pg35-43, Universidade Nove de Julho.

PAIVA, Manoel; Matemática - Paiva/ Manoel- red - São Paulo: Moderna.20o9. 
CHOI, Steve; The Atacama Cosmology Telescope: A Measurement of the Cosmic Microwave Background Power Spectra at 98 and $150 \mathrm{GHz}$

https://scratch.mit.edu/projects/editor/?tutorial=getStarted, acessado em I9/o5/202I, às 20:05.

https://br.pinterest.com/pin/362469470008257343/ , acessado em Io/o5/2021, as I4:30.

http://marcodevilla.com.br/proporcao-e-simetria-dos-dentes-superiores-na-estetica-dosorriso/, acessado em 20/05/2021, às I2:03.

https://iphotochannel.com.br/proporcao-aurea-vs-regra-dos-tercos-qual-e-a-melhor-paracompor-suas-fotos/, acessado em 20/05/2021 as 12:15

https://www.raciociniocristao.com.br/2014/05/digitais-criador/, acessado em 20/05/202I, às I2:17.

https://projetobatente.com.br/regra-aurea-na-arquitetura/ acessado em 15/05/2021, às 13:13 